\title{
Nucleosynthesis of popIII core collapse supernovae and the abundances in extremely metal poor stars
}

\author{
M. Limongi ${ }^{1}$ and A. Chieffi ${ }^{2}$ \\ ${ }^{1}$ INAF - Osservatorio Astronomico di Roma, Via Frascati 33, I-00040 Monteporzio Catone \\ (Roma), ITALY \\ email: marco@mporzio.astro.it \\ ${ }^{2}$ INAF - Istituto di Astrofisica Spaziale e Fisica Cosmica, Via Fosso del Cavaliere, I-00133, \\ Roma, ITALY \\ email: achieffi@rm.iasf.cnr.it
}

\begin{abstract}
We present a new analysis of the abundances observed in extremely metal poor stars based on both a new generation of theoretical presupernova models and explosions of zero metallicity massive stars and a new abundance analysis of an homogeneous sample of stars having $[\mathrm{Fe} / \mathrm{H}] \leqslant-2.5$ (Cayrel et al. 2004).
\end{abstract}

Keywords. Nucleosynthesis, stars: abundances,stars: supernovae: general

\section{Introduction}

Extremely metal poor stars, i.e., those stars having $[\mathrm{Fe} / \mathrm{H}] \leqslant-3.0$, formed in the very early epochs of Galaxy formation hence they reflect the ejecta of the first core collapse supernovae. Wether or not they are associated to single supernovae (Ryan, Norris \& Beers 1996, Audouze \& Silk 1995) or single burst events (Cayrel et al. 2004) they provide very useful constraints to test presupernova models, supernova explosions and nucleosynthesis theories. Moreover the element abundance patterns observed in these stars can be used to infer the nature of the first generations of stars and supernovae.

In a recent paper (Chieffi \& Limongi 2002) we presented a detailed comparison of an extended set of elemental abundances observed in some of the most metal poor stars known at that time and the ejecta produced by a generation of primordial core collapse supernovae. In particular we defined a "template" ultra-metal poor star that represented all the stars in the database and that was compared to the theoretical predictions. Our main findings in that paper were the following: (1) the fit to the "template" star, in particular the $[\mathrm{Si} / \mathrm{Mg}]$ and $[\mathrm{Ca} / \mathrm{Mg}]$ ratios, favored a large carbon abundance at the end of central He burning that, in the framework of classical convection theory, implied a low ${ }^{12} \mathrm{C}(\alpha, \gamma){ }^{16} \mathrm{O}$ cross section - in this case we obtained a good fit to the 8 of the 11 observed element abundance ratios; $(2)$ the fit to $[\mathrm{Sc} / \mathrm{Fe}]$ and $[\mathrm{Co} / \mathrm{Fe}]$ drastically depended on the central $\mathrm{C}$ abundance left by core He burning - at variance with the current beliefs that it is difficult to interpret the observed large overabundance of $[\mathrm{Co} / \mathrm{Fe}]$, a mildly large carbon abundance in the He exhausted core allowed a very good fit to [Co/Fe]; (3) within the grid of models computed at that time it was not possible to find a simultaneous good fit to the remaining three elements, $\mathrm{Ti}, \mathrm{Cr}$ and $\mathrm{Ni}$, even for an arbitrary choice of the mass cut.

The aim of this paper is to present a new analysis of the abundances observed in extremely metal poor stars based on both a new generation of theoretical presupernova 


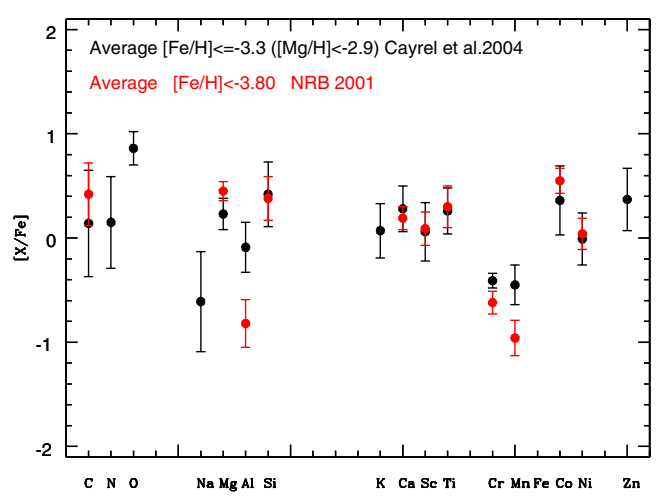

Figure 1. Comparison between average star computed from the Norris, Ryan \& Beers (2001) database (red dots) and that obtained from the Cayrel et al. (2004) database (black dots).

models and explosions of zero metallicity massive stars and a new abundance analysis of an homogeneous sample of stars having $[\mathrm{Fe} / \mathrm{H}] \leqslant-2.5$ (Cayrel et al. 2004).

\section{Theoretical models}

The analysis presented in this paper is based on a new generation of presupernova models and explosions of zero metallicity massive stars. These models, that will be discussed in more detail in a forthcoming paper (Chieffi \& Limongi 2005), have been computed by the latest release (5.050218) of the FRANEC (Frascati RAphson Newton Evolutionary Code) - the previous version has been presented by Limongi \& Chieffi (2003), and references therein.

The main differences with respect to our previous set of models (Chieffi \& Limongi 2002) are the following: the nuclear network is much more extended w.r.t. the previous one and includes now 282 nuclear species, from $\mathrm{H}$ to ${ }^{98} \mathrm{Mo}$, linked by about 3000 reactions; the convective mixing is performed by solving a diffusion equation rather than adopting the Sparks \& Endal (1980) algorithm (see Chieffi, Limongi \& Straniero 1998); the mixing and nuclear burning are fully coupled together and solved simultaneously; updated cross sections have been used whenever possible (see Chieffi \& Limongi 2005 for a full list of references), here we just mention that the cross section for the ${ }^{12} \mathrm{C}(\alpha, \gamma){ }^{16} \mathrm{O}$ is taken from the latest analysis performed by Kunz et al. (2002).

Also the computation of the explosive nucleosynthesis has been greatly improved w.r.t. the previous technique (based on the radiation dominated shock approximation). Indeed, in the present models the explosion has been induced by means of a piston located within the iron core (typically ad $\sim 1 \mathrm{M}_{\odot}$ ) with a given initial velocity and then the passage of the consequent blast wave through the mantle of the star has been followed by means of a PPM hydro code (also this code is presented in detail in Chieffi \& Limongi 2005).

\section{The observable}

The analysis presented in Chieffi \& Limongi (2002) was based on the observations of five stars of metallicity lower than $[\mathrm{Fe} / \mathrm{H}]=-3.3$ (Norris, Ryan \& Beers 2001). Since three of the five stars, i.e., CD $38^{\circ} 245$, CS 22172-002 and CS 22885-096, showed a remarkably similar pattern we could define an "average" (AVG01) star that represented all three of them. Today, a new abundance analysis of a homogeneous sample of 35 stars is available (Cayrel et al. 2004). Within this new database, 22 of the 35 stars have $[\mathrm{Fe} / \mathrm{H}]<-3.0$ and 

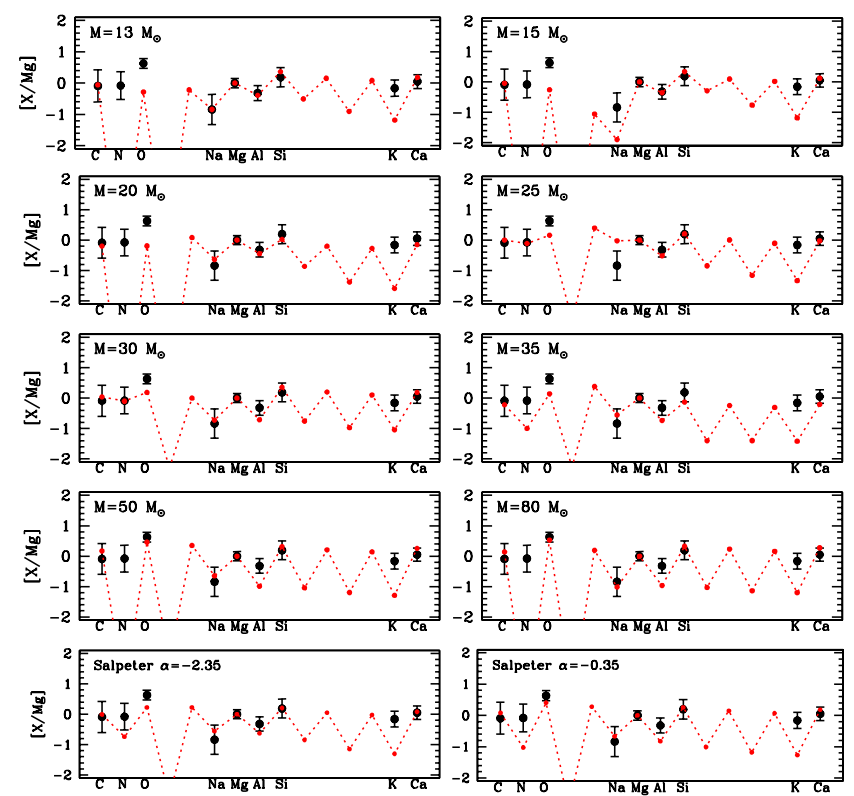

Figure 2. Upper eight panels: comparison between the AVG04 star (black filled dots) and the ejecta of all the models in our grid (red filled dots connected by a dotted line). Lower two panels: comparison between the AVG04 star and the ejecta of all the models in our grid averaged over a Salpeter IMF (red filled dots connected by a dotted line) with two slopes, i.e., $\alpha=-2.35$ and $\alpha=-0.35$.

below $[\mathrm{Mg} / \mathrm{H}]=-3.0$ (which correspond to $[\mathrm{Fe} / \mathrm{H}]=-3.3$ ) the observed element abundance ratios are remarkably uniform, suggesting that the level of "primordial yields" may have been reached. As a consequence, as in Chieffi \& Limongi (2002), we can define an average star (AVG04) that should represent all the stars having $[\mathrm{Fe} / \mathrm{H}]$ lower than -3.3 . A comparison between AVG01 and AVG04 (Fig.1) shows that: (1) more elements are observed for the AVG04 compared to AVG01; (2) AVG04 shows lower values of $[\mathrm{C} / \mathrm{Fe}],[\mathrm{Mg} / \mathrm{Fe}]$ and $[\mathrm{Co} / \mathrm{Fe}]$ and higher values of $[\mathrm{Al} / \mathrm{Fe}],[\mathrm{Cr} / \mathrm{Fe}]$ and $[\mathrm{Mn} / \mathrm{Fe}]$ compared to AVG01 also the relative scaling of these elements are obviously different between the two stars; (3) for each element AVG04 shows larger dispersions compared to AVG01 - this is due to the fact that the average abundances for AVG04 have been obtained with a larger number of stars compared to AVG01.

\section{Fit to light elements (C-Ca)}

Since the elements from $\mathrm{C}$ to $\mathrm{Ca}$ are not affected by the exact location of the mass cut (see Chieffi \& Limongi (2002) for a more details) and since we are interested here in comparing their relative scaling with the observations, it is useful to normalize all their abundances to one of them. We choose to normalize all the abundances of all these elements to Mg. Figure 2 shows the comparison between the AVG04 star and the ejecta of all the models in our grid under the hypothesis that all the models eject some amount of Fe.

The first upper eight panels of Figure 2 clearly shows that the fit to $[\mathrm{C} / \mathrm{Mg}],[\mathrm{Na} / \mathrm{Mg}]$, $[\mathrm{Si} / \mathrm{Mg}]$ and $[\mathrm{Ca} / \mathrm{Mg}]$ is remarkably good for almost all the models without any sizeable 


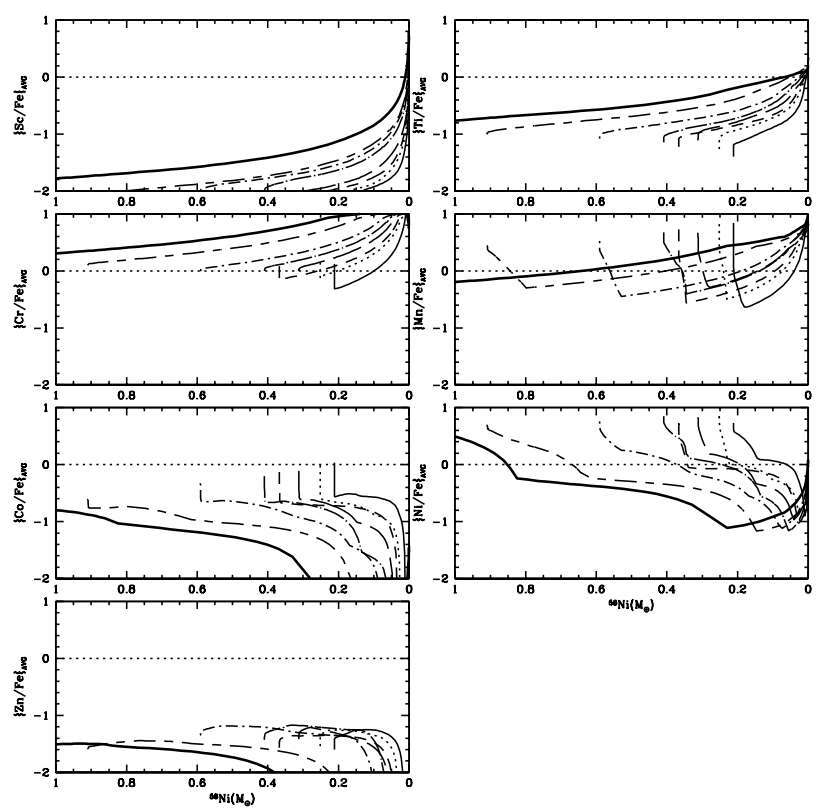

Figure 3. Trends of all the elements produced by the explosive complete and/or incomplete Si burning with the amount of Fe ejected. The eight lines in each panel refer to 13 (solid), 15 (dot), 20 (short dash), 25 (long dash), 30 (dot-short dash), 35 (dot-long dash), 50 (short dash-long dash) and $80 \mathrm{M}_{\odot}$ (tick solid).

dependence on the initial mass. On the contrary the $[\mathrm{O} / \mathrm{Mg}]$ ratio would favour high progenitor masses while $[\mathrm{Al} / \mathrm{Mg}]$ would be better reproduced by low progenitor masses. The $[\mathrm{N} / \mathrm{Mg}] \log$ ratio is very well fitted only by stars in the mass range between 25 and $30 \mathrm{M}_{\odot}$. Such a strong primary $\mathrm{N}$ production is connected, in these stars, with the ingestion of protons by the $\mathrm{He}$ convective shell the penetrates into the $\mathrm{H}$ rich layer. Finally, $[\mathrm{K} / \mathrm{Mg}]$ is always significantly underestimated by all the models. The two lower panels of Figure 2 shows the comparison between the AGV04 star and the ejecta provided by a generation of massive stars (in the mass range 13-80 $M_{\odot}$ ) averaged over a Salpeter IMF for two choices of the slope $\alpha$. In particular, in the standard case $(\alpha=-2.35)$, where the low mass massive stars dominate, $\mathrm{N}$ and $\mathrm{O}$ are both underproduced while $\mathrm{Al}$ is quite well fitted. An opposite behaviour is found when the slope of the IMF is lower ( $\alpha=-$ 0.35 ) and the relative contribution of the more massive stars is higher. In any case $\mathrm{N}$ is significantly underestimated because the high primary $\mathrm{N}$ production occurs in a very narrow mass interval around the $25 \mathrm{M}_{\odot}$.

\section{Fit to the heavy elements (Sc-Zn)}

All the elements between Sc and Zn are produced by either the complete and/or the incomplete explosive Si burning in the deepest layers of the exploding envelope, hence they are those mostly affected by the location of the mass cut. In order to compare the theoretical yields of these elements with their corresponding observations it is useful to introduce the following abundance ratio: $\{\mathrm{X} / \mathrm{Fe}\}_{\text {star }}=\log (\mathrm{X} / \mathrm{Fe})_{\text {model }}-\log (\mathrm{X} / \mathrm{Fe})_{\text {star }}$ (see Chieffi \& Limongi 2002). This ratio is similar to the standard [X/Fe] but in this case the reference star is not the Sun anymore but the star to be fitted. Obviously a perfect fit to the observations is obtained when $\{\mathrm{X} / \mathrm{Fe}\}_{\text {star }}=0$. Figure 3 shows how 

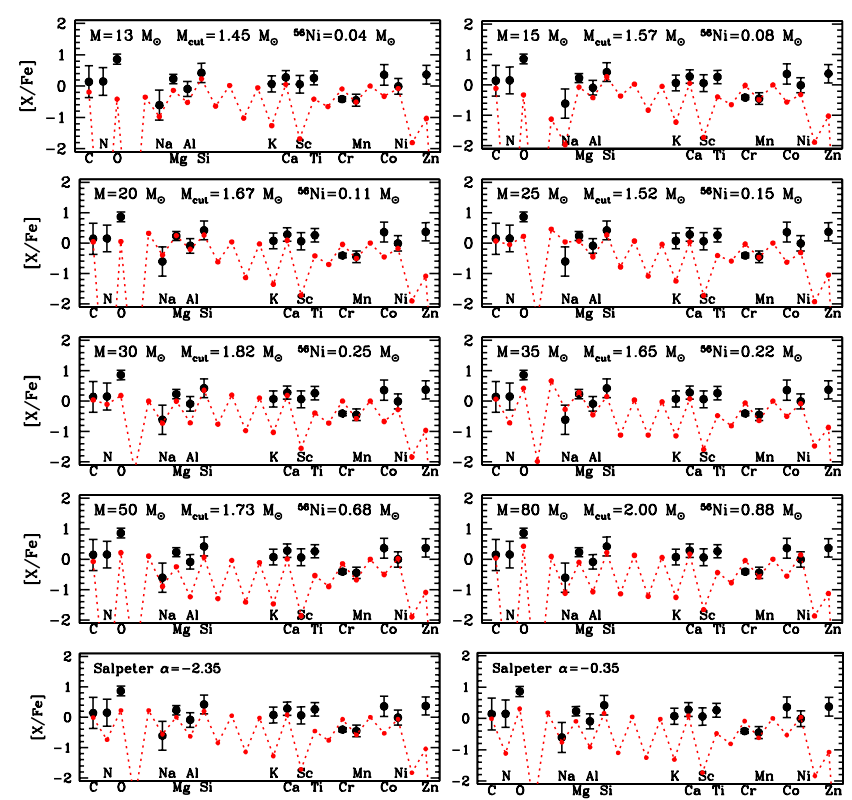

Figure 4. Same as Fig.2 but with a choice of the mass cut (or equivalently ${ }^{56} \mathrm{Ni}$ ), shown in each panel, that provides the best fit to the heavy elements.

$\{\mathrm{Sc}, \mathrm{Ti}, \mathrm{Cr}, \mathrm{Mn}, \mathrm{Co}, \mathrm{Ni}, \mathrm{Zn} / \mathrm{Fe}\}$ vary with the amount of ${ }^{56} \mathrm{Ni}$ ejected (i.e., their dependence on the mass cut) for each stellar model.

From Figure 3 it is clear that there is no value of the mass cut that leads to a simultaneous fit of all the heavy elements from Sc to Zn. In particular, there is no hope of obtaining a good fit to Sc, Ti, Co and Zn for any choice of the mass cut while, on the contrary, there exists a value of ${ }^{56} \mathrm{Ni}$ that would allow a fit to $\mathrm{Cr}, \mathrm{Mn}$ and $\mathrm{Ni}$. In this last case, however, only $\mathrm{Mn}$ and $\mathrm{Ni}$ can be simultaneously fitted by choosing the same value of the mass cut.

Figure 4 shows the best overall fit of all the elements for each stellar model (first upper eight panels) and the best fit obtained by integrating the yields of each model over a Salpeter IMF (two lower panels). Since the amount of Fe required to obtain the best fit to the heavy elements is slightly larger (i.e., a deeper mass cut) than the one needed to fit the light elements, the block of the light elements lowers somewhat without, however, changing their relative scaling (see above). We have shown the best overall fit of all the elements just for sake of completeness, but it is clear that the main problem in the comparison between theory and observations remain the heavy elements $\mathrm{Ti}, \mathrm{Sc}, \mathrm{Cr}, \mathrm{Co}$ and Zn.

While we have no idea about the discrepancy in $[\mathrm{Ti} / \mathrm{Fe}]$ and $[\mathrm{Cr} / \mathrm{Fe}]$, we guess that the fit to $\mathrm{Sc}$, Co and $\mathrm{Zn}$ could be significantly improved by adopting a slightly lower ${ }^{12} \mathrm{C}(\alpha, \gamma){ }^{16} \mathrm{O}$ cross section (still in the range of the experimental uncertainties), as we already have shown in Chieffi \& Limongi (2002). We plan to investigate such a possibility in a forthcoming paper.

An alternative solution that would improve the fit to the heavy elements has been recently proposed by Umeda \& Nomoto (2005). In particular Umeda \& Nomoto (2005) claimed that the overall fit to the heavy elements is greatly improved by means of a more 
energetic explosion, by assuming a mixing-fall back mechanism, by arbitrarily imposing $Y_{e}=0.5001$ in the explosive complete Si burning region and finally by reducing the density of the presupernova model in the innermost zones by a factor of $\sim 3$ w.r.t. the original one. Although a quite good fit can be obtained by arbitrarily changing the properties of the presupernova model and by assuming a more energetic explosion, we like to think that few crucial quantities (like, e.g., the ${ }^{12} \mathrm{C}(\alpha, \gamma){ }^{16} \mathrm{O}$ cross section) can drive the theory towards observations.

\section{Conclusions}

We presented a new comparison between the element abundance ratios observed in extremely metal poor stars $([\mathrm{Fe} / \mathrm{H}]<-3.3$, Cayrel et al. 2004) and our latest yields of zero metallicity core collapse supernovae.

Our main findings are the following: (1) The elements that do not depend on the location of the mass cut (from $\mathrm{C}$ to $\mathrm{Ca}$ ) are quite well reproduced by the models, in particular: $[\mathrm{C}, \mathrm{Na}, \mathrm{Si}, \mathrm{Ca} / \mathrm{Mg}]$ fit pretty well the observations without any significant dependence on the progenitor mass; $[\mathrm{O} / \mathrm{Mg}]$ and $[\mathrm{Al} / \mathrm{Mg}]$ show an anti correlated trend, i.e., $[\mathrm{O} / \mathrm{Fe}]$ would favor high mass progenitors while $[\mathrm{Al} / \mathrm{Mg}]$ is better reproduced by lower mass progenitors; $[\mathrm{N} / \mathrm{Mg}]$ is very well fitted only by stars in the mass range $25-30 \mathrm{M}_{\odot}$, the high primary $\mathrm{N}$ production in these stars being the result of the He convective shell penetration into the $\mathrm{H}$ rich layers; $[\mathrm{K} / \mathrm{Mg}]$ is always significantly underproduced by all the models in the grid. (2) The elements that depend on the location of the mass cut (from Sc to $\mathrm{Zn}$ ) are never simultaneously reproduced by the models for any (unique) choice of the mass cut, $[\mathrm{Mn} / \mathrm{Fe}]$ and $[\mathrm{Ni} / \mathrm{Fe}]$ are the only ones that could be simultaneously fitted for the same value of the mass cut. While we do not have any idea about the discrepancy in $[\mathrm{Ti} / \mathrm{Fe}]$ and $[\mathrm{Cr} / \mathrm{Fe}]$, we suggest that the adoption of a slightly lower ${ }^{12} \mathrm{C}(\alpha, \gamma){ }^{16} \mathrm{O}$ cross section (still in the range of the experimental uncertainties) could significantly improve the fit to the observations of Sc, Co and $\mathrm{Zn}$. We will investigate such a possibility in a forthcoming paper.

\section{Acknowledgements}

I (ML) would like to thank Tatiana Grilli for her continuous encouragement and support during the very hard last year of my life.

\section{References}

Audouze, J., Silk, J. 1995, ApJ (Letters) 451, L49

Cayrel, R., Depagne, E., Spite, M., Hill, V., Spite, F., Fancois, P., Plez, B., Beers, T., Primas, F., Andersen, J., Barbuy, B., Bonifacio, P., Molaro, P., Nordström, B. 2004, A $\& A$ A 416, 1117

Chieffi, A., Limongi, M., Straniero, O. 1998, ApJ 502, 737

Chieffi, A., Limongi, M. 2002, ApJ, 577, 281

Chieffi, A., Limongi, M. 2005, in preparation

Kunz, R., Fey, M., Jaeger, M., Mayer, A., Hammer, J.W., Staudt, G., Harissopulos, S., Paradellis, T. 2002, ApJ 567, 643

Limongi, M., Chieffi, A. 2002, PASA 19, 1

Limongi, M., Chieffi, A. 2003, ApJ 592, 404

Norris, J.E., Ryan, S.G., Beers, T.C. 2001, ApJ 561, 1034

Ryan, S.G., Norris, J.E., Beers, T.C. 1996, ApJ 471, 254

Sparks, W.M., Endal, A.S. 1980, ApJ 237, 130

Umeda, H., Nomoto, K. 2005, ApJ, 619, 427 